\title{
Domain Wall Spacetimes and Particle Motion
}

\author{
Richard Gass and Manash Mukherjee \\ Department of Physics, University of Cincinnati, Cincinnati, OH 45221-0011
}

(September 24, 2018)

\begin{abstract}
We present a mathematical framework for generating thick domain wall solutions to the coupled Einstein-scalar field equations which are (locally) plane symmetric. This approach leads naturally to two broad classes of walllike solutions. The two classes include all previously known thick domain walls. Although one of these classes is static and the other dynamic, the corresponding Einstein-scalar equations share the same mathematical structure independent of the assumption of any reflection symmetry. We also exhibit a class of thick static domain wall spacetimes with different asymptotic vacua. Our analyses of particle motion in such spacetimes raises the interesting possibility that static domain walls will possess a unique experimental signature. 04.20.-q,04.20.Jb
\end{abstract}




\section{INTRODUCTION}

Spontaneous symmetry breaking in the early universe can produce domain walls (sometimes called cosmic membranes). In the simplest model, a domain wall is formed when a discrete symmetry is broken with the resulting discrete set of vacua in different regions of space-time [1]. The study of domain walls has a long history dating back to the work of Zel'dovich et al [2], ( see also [3] for a recent review by Cvetič and Soleng ). Domain walls produced at high temperatures are cosmologically problematic since they produce large-scale anisotropies which violate current experimental bounds [2]: [6]. However, domain walls that are formed after the decoupling of the microwave background do not produce large-scale anisotropies [7]. These late-time domain walls are produced at low temperatures $\left(\sim 10^{-2}\right.$ $\mathrm{eV}$ ) and therefore have characteristic widths on the order of 1 MPc and low-energy densities, thus the name soft domain walls. Soft domain walls have large density gradients and thus are a potential source of the density fluctuations that are necessary for the formation of the large scale structure seen in the universe. It is also interesting to note that the energymomentum tensor for domain walls violates the strong energy condition which results in a gravitational field that is, on average, repulsive [8,9].

In this paper, we consider thick domain walls that arise from a single real scalar field with self-interaction. The associated Einstein-Scalar field equations admit accelerated spherically symmetric (and hence, locally plane symmetric) wall-like solutions. Unlike the previous investigations [8,10] of other thick domain walls, we do not assume any reflection symmetry of the corresponding spacetime, and our analysis of the Einstein equations leads to two broad classes of wall-like solutions which include previously known domain walls. Although non-reflection symmetric domain walls have been previously investigated by Cvetič et al and by Jensen and Soleng [3,11] 15] those walls were thin domain walls. In this paper our interest is in thick domain walls. Furthermore, we find that relaxing the assumption of reflection symmetry leads to a family of static plane symmetric domain walls. This result is consistent with the Dolgov-Khripovich theorem [16]. The Dolgov-Khripovich theorem rules out static 
domain walls provided the vacuum energy density is positive and the metric has the same asymptotic limit on both sides of the wall. By violating the metric condition we are able to

produce explicit solutions for static domain walls. Linet [17] has shown that one can also produce a static wall by using a negative vacuum energy density.

These walls have the unusual property that their asymptotic vacua differ from each other intrinsically.

In section II, we introduce a simple model of domain walls, and discuss the general properties of the associated energy-momentum tensor with particular emphasis on various energy conditions. Without reflection symmetry we obtain, in section III, a new class of domain walls which are (locally) plane symmetric. In section IV, the existence of static domain wall solutions is established. Finally, in section V, we present a detailed analysis of the nontrivial particle motion in domain wall spacetimes. In the Appendix, we prove two lemmas, which are essential for understanding the properties of static and accelerated domain walls.

\section{STRUCTURE OF THE ENERGY MOMENTUM TENSOR}

Following [8] we consider a real self -interacting scalar field on a space-time $\left(M^{4}, g\right)$. The action for the scalar field is given by

$$
\Lambda[\phi]=-\int_{M}\left\{\frac{1}{2} \lambda^{2} \mathbf{d} \phi(\widetilde{\mathbf{d} \phi})+m^{4} V(\phi)\right\} \star \mathbf{1}
$$

where $\lambda$ and $m$ set the energy scales, $\star$ is the Hodge dual operator and the vector field $\widetilde{\mathbf{d} \phi}$ is the metric dual to $\mathbf{d} \phi$. [Throughout this paper, we work with units where $\hbar=c=k_{B}=1$ and $G=\left(1.2 \times 10^{19} \mathrm{GeV}\right)^{-2}$. In these units $\phi$ and the 'potential' $V(\phi)$ are dimensionless.] The stress energy tensor due to $\phi$ is given by

$$
\mathbf{T}=\lambda^{2} \mathbf{d} \phi \otimes \mathbf{d} \phi-\left\{\frac{1}{2} \lambda^{2} \mathbf{d} \phi(\widetilde{\mathbf{d} \phi})+m^{4} V(\phi)\right\} g .
$$

We assume that the potential $V(\phi)$ is positive. This ensures that the energy density

$$
\mathrm{T}(Z, Z)=\frac{\lambda^{2}}{2}\left\{[Z(\phi)]^{2}+\sum_{i=1}^{3}\left[X_{i}(\phi)\right]^{2}\right\}+m^{4} V(\phi)
$$


is positive. Here $\left\{X_{0}, X_{1}, X_{2}, X_{3}\right\}$ form a local orthonormal basis and $Z=X_{0}$ is any observer with $g(Z, Z)=-1$.

Since we are looking for domain wall solutions we require that $V(\phi)$ has two degenerate global minimum at $\phi=\mu_{ \pm}$with $V\left(\mu_{ \pm}\right)=0$. The boundary between these regions is the domain wall where the potential $V(\phi)>0$ and has a maximum at $\phi=\mu_{0}$. The field $\phi$ is a smooth function on spacetime which connects the vacua $\mu_{ \pm}$. Thus, the (ideal) boundary must be a timelike hypersurface in $\left(M^{4}, g\right)$, defined by $\phi=\mu_{0}$. For such field configurations the gradient field, $\widetilde{\mathbf{d} \phi}$, must be normal to the boundary hypersurface, and hence it is spacelike. The stress energy tensor can now be written as

$$
\mathrm{\top}=-\rho(g-\tilde{N} \otimes \tilde{N})+\nu(\tilde{N} \otimes \tilde{N})
$$

where the unit normal $N$ is defined by $N \equiv \widetilde{\mathbf{d} \phi} /[g(\widetilde{\mathbf{d} \phi}, \widetilde{\mathbf{d} \phi})]^{1 / 2}$, and the functions $\rho$ and $\nu$ are given by

$$
\begin{gathered}
\rho=\frac{1}{2} \lambda^{2} \mathbf{d} \phi(\widetilde{\mathbf{d} \phi})+m^{4} V(\phi) \\
\nu=\frac{1}{2} \lambda^{2} \mathbf{d} \phi(\widetilde{\mathbf{d} \phi})-m^{4} V(\phi) .
\end{gathered}
$$

Since $V(\phi) \geq 0$ and $\mathbf{d} \phi(\widetilde{\mathbf{d} \phi})>0$, we have

$$
\begin{array}{r}
\rho>0 \\
\rho+\nu>0
\end{array}
$$

In order to search for a domain wall configuration of the scalar field $\phi$ as a solution to Einstein-Scalar equations

$$
\mathrm{R}_{a b}-\frac{1}{2} \mathrm{R} g_{a b}=(8 \pi G) \mathrm{T}_{a b}
$$

we have made two physically reasonable assumptions:

(i) $V(\phi) \geq 0$

(ii) $\phi$ is spacelike, which means that $g^{a b} \partial_{a} \phi \partial_{b} \phi>0$. 
These two assumptions lead to the following properties of the stress tensor $\mathrm{T}$ :

1. $T_{a b}$ satisfies the weak-energy condition:

For an observer, $u^{a}$ (where $u^{a} u_{a}=-1$ ), we have

$$
u^{a} u^{a} \mathbf{T}_{a b}=\lambda^{2}\left(u^{a} \partial_{a} \phi\right)\left(u^{b} \partial_{b} \phi\right)+\left\{\frac{\lambda^{2}}{2} g^{a b} \partial_{a} \phi \partial_{b} \phi+m^{4} V(\phi)\right\}>0
$$

2. $\mathrm{T}_{a b}$ satisfies the dominant-energy condition:

Defining $v^{a}:=\mathrm{T}^{a b} u_{b}$, we have $v^{a}=\lambda^{2}\left(\partial^{a} \phi\right)\left(\partial^{b} \phi\right) u_{b}-\rho u^{a}$, which implies

$$
v_{a} v^{a}=-\rho^{2}-2 m^{4} \lambda^{2} V(\phi)\left(u_{a} \partial^{a} \phi\right)^{2}<0
$$

3. $\mathrm{T}_{a b}$ violates the strong-energy condition, $u^{a} u^{a} \mathrm{~T}_{a b} \geq-\frac{1}{2} \operatorname{tr}(\mathrm{T})$, for every causal vector $u^{a}$ : To see this we choose an observer $u^{a}$ with $u^{a} u_{a}=-1$ and $u^{a} \partial_{a} \phi=0$. Then, we have

$$
\begin{aligned}
u^{a} u^{a} \mathbf{T}_{a b} & =\left\{\frac{\lambda^{2}}{2} g^{a b} \partial_{a} \phi \partial_{b} \phi+m^{4} \mathbf{V}(\phi)\right\} \\
-\frac{1}{2} \operatorname{tr}(\mathrm{T}) & =\left\{\frac{\lambda^{2}}{2} g^{a b} \partial_{a} \phi \partial_{b} \phi+2 m^{4} \mathbf{V}(\phi)\right\}
\end{aligned}
$$

Hence, $u^{a} u^{a} \mathrm{~T}_{a b}<-\frac{1}{2} \operatorname{tr}(\mathrm{T})$, violating the strong-energy condition for the chosen causal vector field $u^{a}$.

4. Einstein's equations imply the equation of motion for the scalar field $\phi$ :

$$
0=\partial_{a} \phi \mathrm{T}^{a b}{ }_{b}=\left(\partial_{a} \phi \partial^{a} \phi\right)\left\{\lambda^{2} \nabla_{b} \nabla^{b} \phi-m^{4} \frac{d V(\phi)}{d \phi}\right\}
$$

Here, $\left(\partial_{a} \phi \partial^{a} \phi\right) \neq 0$ since $\left(\partial^{a} \phi\right)$ is spacelike, and hence Einstein's equations lead to the scalar-field equation

$$
\lambda^{2} \nabla_{b} \nabla^{b} \phi-m^{4} \frac{d V(\phi)}{d \phi}=0
$$

\section{ACCELERATED SPHERICALLY SYMMETRIC DOMAIN WALLS}

In order to determine the spacetime due to the stress-energy tensor (2.2) we must solve the Einstein equations

$$
\text { Ric }-\frac{1}{2} \mathrm{R} g=8 \pi G \mathrm{~T}
$$


where Ric and R are the Ricci tensor and scalar curvature respectively. To look for a spherically symmetric solution we choose a chart $(t, x, \theta, \psi)$ and assume that the metric admits three Killing vector fields

$$
\begin{aligned}
& \mathrm{K}_{1}=\sin \psi \partial_{\theta}+\cot \theta \cos \psi \partial_{\psi} \\
& \mathrm{K}_{2}=-\cos \psi \partial_{\theta}+\cot \theta \sin \psi \partial_{\psi} \\
& \mathrm{K}_{3}=\partial_{\psi}
\end{aligned}
$$

We consider a spherically symmetric metric of the following form:

$$
g=f^{2}(t, x)\{-\mathbf{d} t \otimes \mathbf{d} t+\mathbf{d} x \otimes \mathbf{d} x\}+h^{2}(t, x)\left\{\mathbf{d} \theta \otimes \mathbf{d} \theta+\sin ^{2} \theta \mathbf{d} \psi \otimes \mathbf{d} \psi\right\}
$$

where the functions $f(t, x)$ and $h(t, x)$ are not assumed to be spatially reflection symmetric. Now, taking Lie-derivatives of Einstein's equations, (3.1), with respect to a Killing vector field $\mathrm{K}$, we find

$$
\begin{array}{r}
\mathrm{L}_{\mathrm{K}} \mathrm{T}=0 \\
\mathrm{~L}_{\mathrm{K}}(\text { trace } \mathrm{T})=0
\end{array}
$$

which, together with the equations (2.4)-(2.6), gives $\mathrm{K}(\rho)=0, \mathrm{~K}(\nu)=0$, and hence $\mathrm{K}(\phi)=$ 0 . Then from (2.2), we have $\partial_{\theta} \phi=\partial_{\psi} \phi=0$. Since $\widetilde{d \phi}$ is spacelike, $\phi$ is assumed to be $t$-independent in the chart $(t, x, \theta, \psi)$. Hence $d \phi=\left(\partial_{x} \phi\right) d x$, and the spacelike unit vector field, $N \equiv \widetilde{d \phi} /[g(\widetilde{d \phi}, \widetilde{d \phi})]^{\frac{1}{2}}$, normal to the membrane is given by $N=(1 / f) \partial_{x}$. Then, the membrane stress tensor $(2.4)$ can be written as

$$
\mathrm{T}=-\rho\left(-e^{0} \otimes e^{0}+e^{2} \otimes e^{2}+e^{3} \otimes e^{3}\right)+\nu e^{1} \otimes e^{1}
$$

where we have defined the coframes by

$$
e^{0}=f d t ; e^{1}=f d x ; e^{2}=h d \theta ; e^{3}=h \sin \theta d \psi
$$

and the corresponding orthonormal frames are

$$
X_{0}=(1 / f) \partial_{t} ; X_{1}=(1 / f) \partial_{x} ; X_{2}=(1 / h) \partial_{\theta} ; X_{3}=(1 / h \sin \theta) \partial_{\psi}
$$


For complete specification of the metric [(3.3)] inside a spacetime region dominated by a thick (cosmic) membrane, it is necessary to solve for the functions $\{f, h\}$ from the Einstein equations

$$
\mathrm{P}_{a}=8 \pi G\left\{\mathrm{~T}_{a b}-(\Gamma / 2) g_{a b}\right\} e^{b}
$$

where $\mathrm{T}$ is given by $(3.5)$, and $\Gamma \equiv \operatorname{trace}[\mathbf{T}]=(-3 \rho+\nu)$. Now, computing the Ricci 1 -forms $\mathrm{P}_{a} \equiv \operatorname{Ric}\left(X_{a}, X_{b}\right) e^{b}$ with respect to the orthonormal basis [(3.6)-(3.7)], we find

$$
\mathrm{P}_{0}=A e^{0}+B e^{1} ; \mathrm{P}_{1}=B e^{0}+C e^{1} ; \mathrm{P}_{2}=D e^{2} ; \mathrm{P}_{3}=D e^{3}
$$

where $\{A, B, C, D\}$ are functions of $\{t, x\}$ [Henceforth, a dot and a prime denote partial differentiations with respect to $t$ and $x]$. Now, from (3.5), (3.8)-(3.9), it is seen that $B=$ $\operatorname{Ric}\left(X_{0}, X_{1}\right)=0$, and hence, in terms of the coeffecients $\{f, h\}$, we have

$$
B=-\left(\frac{2}{f h}\right)\left\{\partial_{x}\left(\frac{\dot{h}}{f}\right)-\left(\frac{\dot{f}}{f^{2}}\right) h^{\prime}\right\}=0
$$

A simple solution to (3.10) can be given by

$$
f \equiv f(x) ; h \equiv c(t) f(x)
$$

Setting $B=0[(3.10)]$ in (3.9) and using (3.11) in the Einstein equations [(3.8)], we find

$$
\begin{aligned}
f^{2} A \equiv \partial_{x}\left(\frac{f^{\prime}}{f}\right)+2\left(\frac{f^{\prime}}{f}\right)^{2}-2\left(\frac{\ddot{c}}{c}\right) & =-4 \pi G(\rho-\nu) f^{2} \\
f^{2} C \equiv-3 \partial_{x}\left(\frac{f^{\prime}}{f}\right) & =4 \pi G(3 \rho+\nu) f^{2} \\
-f^{2} D \equiv \partial_{x}\left(\frac{f^{\prime}}{f}\right)+2\left(\frac{f^{\prime}}{f}\right)^{2}-\left(\frac{\ddot{c}}{c}\right)-\left(\frac{\dot{c}}{c}\right)^{2}-\left(\frac{1}{c}\right)^{2} & =-4 \pi G(\rho-\nu) f^{2}
\end{aligned}
$$

From the first and the third equations in $(3.12)$, we have $(\ddot{c} / c)=\left(1 / c^{2}\right)+(\dot{c} / c)^{2}$ which has a solution

$$
c(t)=\frac{1}{k} \cosh (k t)
$$

where $k>0$. Using (3.13) and defining $u=f^{\prime} / f$, the Einstein equations (3.12) reduce to

$$
2 u^{\prime}+u^{2}-k^{2}=-\left(8 \pi G f^{2}\right) \rho
$$




$$
-3\left(u^{2}-k^{2}\right)=-\left(8 \pi G f^{2}\right) \nu
$$

As a consequence of (3.14)-(3.15) or $\nabla \cdot \mathrm{T}=0$, the equation of motion for the scalar field $\phi$ is given by

$$
\left(\frac{f^{\prime}}{f}\right)=-\frac{1}{3} \frac{\nu^{\prime}}{(\rho+\nu)}
$$

It is clear from (3.5) that $\rho$ and $\nu$ represent energy density and pressure (normal to the wall) with respect to the orthonormal basis in (3.6)-(3.7) : $\mathrm{T}\left(X_{0}, X_{0}\right)=\rho ; \mathrm{T}\left(X_{1}, X_{1}\right)=\nu$, and by (3.14)-(3.15), $\rho \equiv \rho(x)$ and $\nu \equiv \nu(x)$ in the chart $\{t, x, y, z\}$. [Here we remark that components of the stress tensor [(3.5)] are not, in general, time-independent with respect to the coordinate basis. For example, $\mathrm{T}\left(\partial_{y}, \partial_{y}\right)=-\rho \exp (k t)$ by (3.5),(3.11) and (3.13)]. Furthermore, $\nu=0$ implies $\left(f^{\prime} / f\right)^{2}=k^{2}$, and hence $\rho=0$ - leading to an empty spacetime (vacuum). Thus the pressure, $(\nu)$, inside the membranes can not be zero. We now show that the nowhere zero smooth function $\nu(x)<0$ based on the constraint $(\rho+\nu)>0$, and additional physical assumptions that $f$ is bounded and nowhere zero, and the stress tensor [(3.5)] tends to zero in the limit $|x| \rightarrow \infty$ giving rise to asymptotic vacua.

\subsection{Negative 'Pressure' $(\nu)$ :}

From equations (3.12) and (3.14)-(3.16), we have

$$
\begin{aligned}
3 u^{\prime} & =-4 \pi G(3 \rho+\nu) f^{2} \\
\nu^{\prime} & =-3(\rho+\nu) u
\end{aligned}
$$

From the equation (3.17), it follows that $u^{\prime}(x)<0$, and hence, $u$ has at most one zero. Also, for the nowhere zero smooth function, $\nu$, there exists an $\bar{x}$ such that $\nu^{\prime}(\bar{x})=0$ (See Appendix, Lemma 2, for a proof of this statement). Then, (3.18) implies that each of the functions $u$ and $\nu^{\prime}$ has a unique zero at $x=\bar{x}$. Furthermore, differentiating (3.18) and using (3.17), we have $\nu^{\prime \prime}(\bar{x})=-(\rho+\nu) u^{\prime}(\bar{x})>0$ - which means that the unique extremum of $\nu$ over $\mathbb{R}$ is a minimum at $x=\bar{x}$. However, $\nu$ satisfies the asymptotic condition $\lim _{|x| \rightarrow \infty} \nu=0$, and hence $\nu$ must be negative. As a consequence of $\nu<0$, we have $\rho>|\nu|$ since $\rho>0$ and $(\rho+\nu)>0$. 


\subsection{The Equation of State ' $\nu \equiv \nu(\rho)^{\prime}$ :}

In order to solve the Einstein-scalar equations (3.14)-(3.15), we need an equation of state $\nu \equiv \nu(\rho)$ subject to the constraints $\nu<0, \rho>|\nu|, \lim _{|x| \rightarrow \infty} \nu=0$ and $\lim _{|x| \rightarrow \infty} \rho=0$. First, we observe that $u^{\prime}(x)<0 \forall x$, and hence, from (3.15), $\lim _{x \rightarrow \pm \infty} u(x)=\mp k$. From these properties of $u(x)$, it follows that the non-zero smooth functions $\left(u^{2}-k^{2}\right)$ and $u^{\prime}$ are both negative; each of them has a unique minimum and tends to zero in the limit $|x| \rightarrow \infty$. Using (3.14) and (3.15), we also have

$$
\frac{u^{\prime}(x)}{k^{2}-u(x)^{2}}=-\epsilon(x)
$$

where the nowhere zero smooth function $\epsilon(x)$ is given by

$$
\epsilon(x)=\frac{1}{2}\left[\frac{3 \rho(x)}{|\nu(x)|}-1\right]>1
$$

since $\rho(x)>|\nu(x)|$. In fact, the equations (3.19) and (3.20) lead to an equation of state $\nu=-v_{0}^{2} \rho$ where $\left(3-v_{0}^{2}\right) / 2 v_{0}^{2}=\epsilon(x)>1$, and hence, $v_{0}^{2}<1$. It is important to note that $\epsilon(x)$ is dimensionless according to our choice of units [specified at the beginning of section II]. Thus, $\epsilon(x)$ is, in fact, a function of the dimensionless argument, $k x$, where the dimension of $k$ is given by inverse of length.

To determine the classes of admissible $\epsilon(x)$ leading to domain wall configurations of $\phi(x)$, we recall from section II [and the discussion below (3.3)] that $\lim _{x \rightarrow \pm \infty} \phi(x)=\mu_{ \pm}$, where the constants $\mu_{+}$and $\mu_{-}$represent two degenerate vacua with $V\left(\mu_{ \pm}\right)=0$. However, from

the equations (2.5)-(2.6), (3.3) and (3.14)-(3.15), and the existence of asymptotic limits of $\phi$, we have

$$
\begin{aligned}
\phi^{\prime}(x)^{2} & =\left(2 / \lambda^{2} 8 \pi G\right)[\epsilon(x)-1]\left[k^{2}-u(x)^{2}\right] \\
\lim _{|x| \rightarrow \infty} \phi^{\prime}(x) & =0
\end{aligned}
$$

where $\lim _{|x| \rightarrow \infty}\left[k^{2}-u(x)^{2}\right]=0$. Thus, any $\epsilon(x)$ from the class of bounded smooth functions such that $1<\epsilon(x) \leq \epsilon_{0}$, will satisfy the condition (3.21).

One can also choose $\epsilon(x)$ to be unbounded. In this case, we have another family of admissible smooth functions satisfying (3.21) provided, asymptotically, $\left[k^{2}-u(x)^{2}\right]$ approches 
zero faster than $\epsilon(x)$ tends to infinity. To see this, we integrate (3.19) to obtain $u(x)$ :

$$
u(x)=-k \tanh \left(k \int \epsilon(x) d x+c\right)
$$

where $c$ is an integration constant. Inserting the above expression for $u(x)$ in $\left[k^{2}-u(x)^{2}\right]$, we find

$$
\left[k^{2}-u(x)^{2}\right]=k^{2} \operatorname{sech}^{2}\left(k \int \epsilon(x) d x+c\right)
$$

The first equation in (3.21) now gives

$$
\phi^{\prime}(x)^{2}=\left(2 k^{2} / \lambda^{2} 8 \pi G\right)[\epsilon(x)-1] \operatorname{sech}^{2}\left(k \int \epsilon(x) d x+c\right)
$$

Since $\operatorname{sech}^{2}\left(k \int \epsilon(x) d x+c\right)$ tends to zero exponentially as $|x| \rightarrow \infty$, we may take $\epsilon(x)$ to be, for example, a polynomial such as $2+3 k^{2} x^{2}$ for which (3.20) and (3.21) are satisfied.

This suggests a procedure for solving the Einstein equations, (3.14)-(3.15), with a choice of $\epsilon(x)$ from these two large classes of functions leading to domain walls. In the following subsection, we will discuss solutions due to bounded $\epsilon(x)$.

\subsection{Solutions to the Einstein Equations:}

A simple solution to the Einstein equations, (3.14) and (3.15), is obtained by choosing $\epsilon(x) \equiv \epsilon_{0}>1$ - which trivially belongs to the class of smooth bounded functions. Hence, such a choice ensures, through (3.21), the existence of a domain wall solution to the Einstein equations. Integrating (3.19) and using $u \equiv f^{\prime} / f$, we find

$$
\begin{aligned}
& u(x)=-k \tanh \left(\epsilon_{0} k x\right) \\
& f(x)=\cosh ^{-q}(k x / q)
\end{aligned}
$$

where $q \equiv 1 / \epsilon_{0}$. Also, from (3.21) and (3.22), we have the following exact solutions for $\phi$ and $V(\phi)$ :

$$
\begin{aligned}
\phi & =\arctan [\sinh (k x / q)] \\
V(\phi) & =\{\cos \phi\}^{2(1-q)}
\end{aligned}
$$


where the parameters $q$ and $k$ are related to the energy scales $\lambda$ and $m$ by

$$
\begin{aligned}
\lambda^{2} & =2 q(1-q)(1 / 8 \pi G) \\
m^{4} & =k^{2}\left(\frac{1}{q}+2\right)(1 / 8 \pi G)
\end{aligned}
$$

Thus, $f(x), \phi(x)$ and $V(\phi)$ solve the Einstein equations as well as the field equation for the real scalar $\phi$. Finally, the metric for the accelerated spherically symmetric domain wall spacetime is given by

$$
g=f^{2}(x)\{-\mathbf{d} t \otimes \mathbf{d} t+\mathbf{d} x \otimes \mathbf{d} x\}+\left(\frac{1}{k} \cosh (k t)\right)^{2} f^{2}(x)\left\{\mathbf{d} \theta \otimes \mathbf{d} \theta+\sin ^{2} \theta \mathbf{d} \psi \otimes \mathbf{d} \psi\right\}
$$

It is interesting to note that for a fixed $x=x_{0}$, the spherical domain represented by (a 3-dimensional De-Sitter slice)

$$
\left.g\right|_{x=x_{0}}=f^{2}\left(x_{0}\right)\left\{-\mathbf{d} t \otimes \mathbf{d} t+\left(\frac{1}{k} \cosh (k t)\right)^{2}\left(\mathbf{d} \theta \otimes \mathbf{d} \theta+\sin ^{2} \theta \mathbf{d} \psi \otimes \mathbf{d} \psi\right)\right\}
$$

first contracts and then expands. Furthermore, if we choose a chart $\{\tau, \eta, y, z\}$ with the following (implicit) coordinate transformations

$$
\begin{aligned}
& \tau=\left(\frac{1}{k}\right) \ln \left(\frac{1}{k} \sinh k t+\frac{1}{k} \cosh k t \cos \theta\right) \\
& \eta=x \\
& y=(\cosh k t \sin \theta \cos \psi) /(\sinh k t+\cosh k t \cos \theta) \\
& z=(\cosh k t \sin \theta \sin \psi) /(\sinh k t+\cosh k t \cos \theta)
\end{aligned}
$$

the wall spacetime (3.23) becomes (locally) plane symmetric:

$$
g=f^{2}(\eta)\{-\mathbf{d} \tau \otimes \mathbf{d} \tau+\mathbf{d} \eta \otimes \mathbf{d} \eta\}+\exp (2 k \tau) f^{2}(\eta)\{\mathbf{d} y \otimes \mathbf{d} y+\mathbf{d} z \otimes \mathbf{d} z\}
$$

The metric (3.23) or (3.26) is only one of many possible domain wall solutions and the equations (3.19)-(3.20) show that admissible choices for $\epsilon(x)$ generate additional solutions. For example, the smooth function $\epsilon(x)=2\left[1-k x \exp \left(-k^{2} x^{2}\right)\right]>1$ has aymptotic limits, 
$\lim _{|x| \rightarrow \infty} \epsilon(x)=2$. Thus, $\epsilon(x)$ belongs to the class of bounded smooth functions so that (3.21) is satisfied, and hence, $\epsilon(x)$ must lead to a domain wall solution to the Einstein equations.

The equation (3.19) for $u(x)$ then becomes

$$
\frac{u^{\prime}(x)}{k^{2}-u(x)^{2}}=-2\left[1-k x \exp \left(-k^{2} x^{2}\right)\right] \text {. }
$$

By integrating (3.27), we get

$$
u(x)=-k \tanh \left[2 k x+\exp \left(-k^{2} x^{2}\right)-C\right]
$$

where $\mathrm{C}$ is a constant of integration which we will set to zero. The constant $C$ determines where $u(x)$ crosses the x-axis. By adjusting $C$ the function $u(x)$ can be made to cross the $\mathrm{x}$-axis at the origin. Since $u=f^{\prime} / f$ we can now solve for $f(x)$ which gives

$$
f(x)=\exp \left[-k \int \tanh \left(2 k x+\exp \left(-k^{2} x^{2}\right)\right) d x\right]
$$

The integral in (3.29) which can not be evaluated analytically, is easily done numerically. A plot of $f(x)$ is shown in Fig 1 .

The energy density $\rho(x)$ and the pressure $\nu(x)$ can be computed from the Einstein equations (3.14) and (3.15) and are shown in Fig 2 and Fig 3.The equation of state can no longer be found analytically but it is possible to parametricaly plot the energy density and the pressure as a function of position. This is shown in Fig 4. The non-zero area inclosed by the $\rho-\nu$ diagram is due to the lack of reflection symmetry in the stress tensor. The kink in the curve is due to the bump in the potential $V(\phi)$ which is shown in figure 5 . The field $\phi$ which generates $V(\phi)$ is shown in figure 6 .

The equations (3.19)-(3.20) clearly allow one to generate two broad classes of accelerated spherically symmetric domain wall spacetimes but all of these solutions lead to solition-like configurations for the scalar field $\phi$ and have geodesics with the same general features. These solutions may or may not be reflection symmetric in $x$-coordinate. In the next section, we will show that static wall spacetimes cannot be reflection symmetric, leading to non-trivial asymptotic vacuum structures. 


\section{STATIC PLANE SYMMETRIC DOMAIN WALLS}

We now look for static solutions to the Einstein-Scalar equations. Imposing plane symmetry, the metric for a static spacetime takes the following form [18]:

$$
g=f^{2}(x)\{-d t \otimes d t+d x \otimes d x\}+h^{2}(x)\{d y \otimes d y+d z \otimes d z\}
$$

Then the Einstein's equations corresponding to the domain wall stress tensor are given by

$$
\begin{gathered}
u^{\prime}+2 u v=-(4 \pi G) f^{2}(\rho-\nu), \\
u^{\prime}+2\left(v^{\prime}+v^{2}-u v\right)=-(4 \pi G) f^{2}(3 \rho+\nu) \\
v^{\prime}+2 v^{2}=-(4 \pi G) f^{2}(\rho-\nu)
\end{gathered}
$$

where, we set $u \equiv\left(f^{\prime} / f\right)$ and $v \equiv\left(h^{\prime} / h\right)$. Equations (4.2)-(4.4) also imply [see Appendix]

$$
\begin{gathered}
\left(u^{\prime}+2 v^{\prime}\right)<0 \\
\nu^{\prime}=-(\rho+\nu)(u+2 v)
\end{gathered}
$$

The above equations lead to a number of general properties of the plane symmetric static domain wall, assuming that $(\rho+\nu)>0, \lim _{|x| \rightarrow \infty} \nu=0$ and $\lim _{|x| \rightarrow \infty} \rho=0$.

\subsection{Negative 'Pressure' $(\nu)$ :}

From the equation (4.5), it follows that $(u+2 v)$ has at most one zero. Also, for a nowhere zero smooth function, $\nu$, there exists an $\bar{x}$ such that $\nu^{\prime}(\bar{x})=0$ (See Appendix, Lemma 2, for a proof of this statement). Then, (4.6) implies that each of the functions $(u+2 v)$ and $\nu^{\prime}$ has a unique zero at $x=\bar{x}$. Furthermore, differentiating (4.6) and using (4.5), we have 
$\nu^{\prime \prime}(\bar{x})=-(\rho+\nu)\left[u^{\prime}(\bar{x})+2 v^{\prime}(\bar{x})\right]>0$ - which means that the unique extremum of $\nu$ over $\mathbb{R}$ is a minimum at $x=\bar{x}$. However, $\nu$ satisfies the asymptotic condition $\lim _{|x| \rightarrow \infty} \nu=0$, and hence $\nu$ must be negative.

\subsection{Plane Symmetric Static Wall is Not Reflection Symmetric :}

If the metric (4.1) is reflection symmetric about $x=0$ (say) then $f(x)=f(-x)$ and hence, $f^{\prime}(x)=-f^{\prime}(-x)$ and $f^{\prime}(0)=0$. Similar results hold for $h(x)$, and $u(0)=0=v(0)$. Also, from the Einstein's equations (4.2) and (4.3), we have

$$
\left(v^{\prime}-u^{\prime}\right)+2 v(v-u)=0
$$

Integrating once gives,

$$
[v(x)-u(x)] h^{2}(x)=K / 2
$$

for all $\mathrm{x}$ where, $K$ is a constant. For $K \neq 0$, equation (4.7) contradicts reflection symmetry of $f(x)$ and $h(x)$, and hence a plane symmetric static wall can not have reflection symmetry. If $K=0, h(x)$ is proportional to $f(x)$ and with the linear changes in coordinates we have $f(x)=h(x)$ and $u(x)=v(x)$. Then from Einstein's equations,

$$
\begin{gathered}
u^{\prime}+2 u^{2}=-(4 \pi G) f^{2}(\rho-\nu) \\
u^{\prime}+2\left(v^{\prime}+v^{2}-u v\right)=-(4 \pi G) f^{2}(3 \rho+\nu)
\end{gathered}
$$

we find

$$
0 \leq 3 u^{2}=(8 \pi G) f^{2} \nu \leq 0
$$

since, $\nu<0$ for non-zero $\nu$. Thus, for $K=0$ we have $\nu=0$, and hence $\rho=0$ producing a vacuum. This conclusion also means that a conformally flat plane symmetric static metric leads to a vacuum spacetime under the asymptotic conditions on $\nu$ and $\rho$. 


\subsection{Asymptotic Limits of The Einstein Equations :}

If we assume, again, that pressure $(\nu)$ and energy density $(\rho)$ vanish in the region far from a thick membrane, then the following equations

$$
\lim _{|x| \rightarrow \infty} \nu=0 ; \lim _{|x| \rightarrow \infty} \rho=0
$$

generate the vacuum Einstein's equations:

$$
\begin{gathered}
u^{\prime}+2 u v=0 \\
u^{\prime}+2\left(v^{\prime}+v^{2}-u v\right)=0 \\
v^{\prime}+2 v^{2}=0
\end{gathered}
$$

From these equations, we find an algebraic relation

$$
(2 u+v) v=0
$$

(a) If $\left(h^{\prime} / h\right) \equiv v=0$, then $\left(f^{\prime} / f\right)^{\prime} \equiv u^{\prime}=0$. In this case, we may take $h(x)=1$, and on integration $f(x)=\exp (p x)$, where $p$ is a constant. Inserting the following coordinate transformations

$$
\tilde{t}=\frac{1}{p} \exp (p t) \sinh (p x) ; \tilde{x}=\frac{1}{p} \exp (p t) \cosh (p x) ; \tilde{y}=y ; \tilde{z}=z
$$

in (4.1), we have the Minkowski spacetime:

$$
g_{\text {Mink }}=-d \tilde{t} \otimes d \tilde{t}+d \tilde{x} \otimes d \tilde{x}+d \tilde{y} \otimes d \tilde{y}+d \tilde{z} \otimes d \tilde{z}
$$

(b) If $2\left(f^{\prime} / f\right)+\left(h^{\prime} / h\right) \equiv 2 u+v=0$, by integrating once we have $f^{2}(x) h(x)=C_{0}$, where $C_{0}$ is a constant. Also, (4.10) implies $h^{2}(x)=x$, and choosing $C_{0}=1$ we get $f^{2}(x)=1 / \sqrt{x}$. In 
this case, we have Taub's plane symmetric vaccum spacetime [18]:

$$
g_{\text {Taub }}=\frac{1}{\sqrt{x}}\{-d t \otimes d t+d x \otimes d x\}+x\{d y \otimes d y+d z \otimes d z\}
$$

\subsection{Solutions to Einstein's Equations :}

In order to obtain a plane symmetric static wall solution to Einstein's equations (4.2), (4.3) and (4.4) we now introduce a coordinate transformation suggested by the equation (4.7) :

$$
\frac{1}{h(x)} \frac{d h(x)}{d x}=\frac{1}{f(x)} \frac{d f(x)}{d x}+\frac{K}{2 h^{2}(x)}
$$

If we define the non-singular coordinate change $\xi \equiv \xi(x)$ by

$$
d \xi=\frac{d x}{h^{2}(x)}
$$

then considering $f(x)$ and $h(x)$ as functions of $\xi$, we have

$$
\frac{1}{h} \frac{d h}{d \xi}=\frac{1}{f} \frac{d f}{d \xi}+\frac{K}{2}
$$

Since $f(x) d x=f(x(\xi)) h^{2}(x(\xi)) d \xi$, we define

$$
f(x(\xi)) \equiv F(\xi) \text { and } N(\xi) \equiv f(x(\xi)) h^{2}(x(\xi))
$$

Thus, $h^{2}(x(\xi))=\frac{N(\xi)}{F(\xi)}$, and hence, in the new coordinate system $\{t, \xi, y, z\}$, the plane symmetric static metric (4.1) takes the following form:

$$
g=-F^{2}(\xi) d t \otimes d t+N^{2}(\xi) d \xi \otimes d \xi+\frac{N(\xi)}{F(\xi)}\{d y \otimes d y+d z \otimes d z\}
$$

Denoting differentiation with respect to $\xi$ by a $\left(^{\prime}\right)$, the equation (4.13) implies

$$
\left(\frac{N^{\prime}}{N}\right)=3\left(\frac{F^{\prime}}{F}\right)+K
$$

Einstein's equations corresponding to the metric (4.15) are given by 


$$
\begin{array}{r}
\left(\frac{F^{\prime}}{F}\right)^{\prime}=-(4 \pi G) N^{2}(\rho-\nu) \\
\frac{3}{2}\left(\frac{F^{\prime}}{F}\right)^{2}-\left(\frac{F^{\prime}}{F}\right)\left(\frac{N^{\prime}}{N}\right)-\frac{1}{2}\left(\frac{N^{\prime}}{N}\right)^{2}+\left(\frac{N^{\prime}}{N}\right)^{\prime}=-(4 \pi G) N^{2}(3 \rho+\nu) \\
\frac{1}{2}\left\{\left(\frac{N^{\prime}}{N}\right)^{\prime}-\left(\frac{F^{\prime}}{F}\right)^{\prime}\right\}=-(4 \pi G) N^{2}(\rho-\nu)
\end{array}
$$

From (4.17)-(4.19) and (4.16), we have

$$
\begin{aligned}
-2\left(\frac{F^{\prime}}{F}\right)^{\prime}+ & \left\{3\left(\frac{F^{\prime}}{F}\right)^{2}+2\left(\frac{F^{\prime}}{F}\right) K+K^{2} / 4\right\}=(8 \pi G) N^{2} \rho(\xi) \\
& \left\{3\left(\frac{F^{\prime}}{F}\right)^{2}+2\left(\frac{F^{\prime}}{F}\right) K+K^{2} / 4\right\}=(8 \pi G) N^{2} \nu(\xi)
\end{aligned}
$$

If we set $r(\xi) \equiv F^{\prime}(\xi) / F(\xi)+K / 3$ and $\alpha \equiv K / 6$, then the Einstein equations (4.20) becomes

$$
\begin{aligned}
2 r^{\prime}-3\left(r^{2}-\alpha^{2}\right) & =-\left(8 \pi G N^{2}\right) \rho \\
-3\left(r^{2}-\alpha^{2}\right) & =-\left(8 \pi G N^{2}\right) \nu
\end{aligned}
$$

From this form of the Einstein equations, we have

$$
\frac{r^{\prime}(\xi)}{\alpha^{2}-r(\xi)^{2}}=-\sigma(\xi)
$$

where the nowhere zero smooth function $\sigma(\xi)$ is given by

$$
\sigma(\xi)=\frac{3}{2}\left[\frac{3 \rho(\xi)}{|\nu(\xi)|}+1\right]>3
$$

since $\rho(\xi)>|\nu(\xi)|$. Since $\nu<0$, we have an equation of state in the form $\nu(\xi)=-q(\xi) \rho(\xi)$, where the relation $\frac{3}{2}\left[\frac{3 \rho(\xi)}{|\nu(\xi)|}+1\right]=\sigma(\xi)>3$ is equivalent to $0<q(\xi)<1$. Following 
the procedure developed in subsection 3.2, for generating solutions to the Einstein-scalar equations, we obtain the simplest static domain wall solution to (4.20)-(4.21) when $q$ is a constant :

$$
\ln F=-\frac{L}{3} \ln \left[\cosh \left(\frac{K \xi}{2 L}\right)\right]-\frac{K \xi}{3}
$$

where $L$ is related to $q$ by $q=L /(2-L)$ and hence, $0<L<1$. Now, using the equation (4.16) we find the metric functions:

$$
\begin{gathered}
F^{2}=(\cosh (K \xi / 2 L))^{-\frac{2 L}{3}} \exp \left(-\frac{2 K \xi}{3}\right) \\
N^{2}=(\cosh (K \xi / 2 L))^{-2 L} \\
\left(\frac{N}{F}\right)=(\cosh (K \xi / 2 L))^{-\frac{2 L}{3}} \exp \left(\frac{K \xi}{3}\right)
\end{gathered}
$$

Equations (4.25)-(4.27) represent a two-parameter $(K, L)$ family of plane symmetric static spacetimes dominated by domain walls. The 'density' $(\rho)$ and 'pressure' $(\nu)$ functions are obtained from the Einstein's equations [4.20-4.21]:

$$
\begin{gathered}
\rho(\xi)=\frac{K^{2}(2-L) \operatorname{sech}\left(\frac{K \xi}{2 L}\right)^{2-2 L}}{96 G L \pi} \\
\nu(\xi)=\frac{-K^{2} \operatorname{sech}\left(\frac{K \xi}{2 L}\right)^{2-2 L}}{96 G \pi}
\end{gathered}
$$

The explicit forms of the (dimensionless) scalar field and the potential are given by

$$
\begin{gathered}
\phi(\xi)=\arctan \left(\sinh \left(\frac{K \xi}{2 L}\right)\right) \\
V(\phi)=(\cos \phi)^{2-2 L}
\end{gathered}
$$


The energy scales, $\lambda$ and $m$, are related to the parameters, $K$ and $L$, by

$$
\begin{aligned}
& \lambda^{2}=\frac{(1-L) L}{12 G \pi} \\
& m^{4}=\frac{K^{2}}{48 G L \pi}
\end{aligned}
$$

It should be noted from (4.28)-(4.29) that the matter density and pressure are reflection symmetric, while the spacetime [determined by (4.25)-(4.27)] is not. The absence of reflection symmetry of the static wall spacetime is also demonstrated by the existence of different asymptotic vacua defined by

$$
\lim _{|\xi| \rightarrow \infty} \nu=0 ; \lim _{|\xi| \rightarrow \infty} \rho=0
$$

For $\xi \rightarrow \infty$,

$$
\begin{aligned}
F^{2} & \rightarrow \exp (-K \xi) \\
N^{2} & \rightarrow \exp (-K \xi) \\
\left(\frac{N}{F}\right) & \rightarrow \text { constant }
\end{aligned}
$$

Comparing our results in subection 4.3, it is clear that the limits (4.34)-(4.36) implies a Minkowski vacuum. For $\xi \rightarrow-\infty$,

$$
\begin{aligned}
F^{2} & \rightarrow \exp \left(-\frac{1}{3} K \xi\right) \\
N^{2} & \rightarrow \exp (K \xi) \\
\left(\frac{N}{F}\right) & \rightarrow \exp \left(\frac{2}{3} K \xi\right)
\end{aligned}
$$

The fact that the limits (4.37)-(4.39) yields the Taub vacuum, can be seen as follow:

$$
g_{\xi \rightarrow-\infty}=-\exp \left(-\frac{1}{3} K \xi\right) d t \otimes d t+\exp (K \xi) d \xi \otimes d \xi+\exp \left(\frac{2}{3} K \xi\right)\{d y \otimes d y+d z \otimes d z\}
$$


Then, the coordinate transformations given by

$$
\begin{aligned}
& t=(2 K / 3)^{\frac{1}{3}} t_{o} \\
& \xi=(3 / 2 K) \ln \left\{(2 K / 3)^{\frac{4}{3}} \xi_{o}\right\} \\
& y=(3 / 2 K)^{\frac{2}{3}} y_{o} \\
& z=(3 / 2 K)^{\frac{2}{3}} z_{o}
\end{aligned}
$$

lead to the metric for the Taub vacuum:

$$
g_{\text {Taub }}=\frac{1}{\sqrt{\xi_{o}}}\left\{-d t_{o} \otimes d t_{o}+d \xi_{o} \otimes d \xi_{o}\right\}+\xi_{o}\left\{d y_{o} \otimes d y_{o}+d z_{o} \otimes d z_{o}\right\}
$$

\subsection{More Static Solutions :}

In $(4.22)$, if we take a nowhere zero bounded smooth function

$$
\sigma(\xi)=9\left[1+\exp \left(-36 \alpha^{2} \xi^{2}\right)\right]
$$

which satisfies (4.23), then we have

$$
r(\xi)=-\alpha \tanh \left[(9 \alpha) \xi+\left(\frac{3}{4}\right) \sqrt{\pi} \operatorname{Erf}(6 \alpha \xi)-C\right]
$$

A plot of $r(\xi)$ is shown, for $C=0$ and $\alpha=1 / 6$ [or equivalently, $K=1$ ], in figure 7 . The function $F(\xi)$ can be found by numerical integration. A plot of $F(\xi)$ is shown in figure 8 . Equation 4.16 can be integrated to give $N(\xi)$. A plot of $N(\xi)$ is shown in figure 9 .

\section{PARTICLE MOTION}

For domain wall spacetimes gravity is, on average, repulsive. This can be seen from the following argument. The condition, $\operatorname{Ric}(v, v) \geq 0$ for all observers $v$, expresses the 
emperical fact that, on average, gravity attracts [19]. This condition is equivalent to the strong energy condition, $\mathrm{T}(v, v) \geq-\frac{\Gamma}{2}$ for all observers $v$, where $\Gamma=\operatorname{trace}(\mathbf{T})$. Thus, violation of the strong energy condition for the Einstein-Scalar stress tensor implies that, on average, gravity is repulsive in the domain wall spacetime.

\section{A. Accelerated Spacetimes}

To obtain the geodesic eqations for a particle (of mass $m$ ) in the accelerated domain wall spacetime [equation (3.23)], we consider its worldline in the equatorial plane $[\theta=\pi / 2]$ :

$$
\gamma: \tau \mapsto(t=t(\tau), x=(\tau), \theta=\pi / 2, \psi=\psi(\tau))
$$

If $\gamma$ is a geodesic (and $\tau$ is an affine parameter along $\gamma$ ), then the 4-velocity of the particle is given by

$$
\gamma_{*}=\left(\frac{d t}{d \tau}\right) \partial_{t}+\left(\frac{d x}{d \tau}\right) \partial_{x}+\left(\frac{d \psi}{d \tau}\right) \partial_{\psi}
$$

with the normalisation $g\left(\gamma_{*}, \gamma_{*}\right)=-m^{2}$ :

$$
-f^{2}\left(\frac{d t}{d \tau}\right)^{2}+f^{2}\left(\frac{d x}{d \tau}\right)^{2}+f^{2}(\cosh k t / k)^{2}\left(\frac{d \psi}{d \tau}\right)^{2}=-m^{2}
$$

Then the geodesic $\gamma$ is specified by

$$
\begin{aligned}
\left(\frac{d x}{d \tau}\right)^{2} & =\left(\frac{1}{f^{2}}\right)\left(\frac{a_{0}}{f^{2}}-m^{2}\right) \\
\left(\frac{d t}{d \tau}\right)^{2} & =\left(\frac{1}{f^{2}}\right)\left\{a_{0}-\left(\frac{a_{3} k}{\cosh k t}\right)^{2}\right\} \\
\frac{d \psi}{d \tau} & =\left(\frac{1}{f^{2}}\right)\left(\frac{a_{3} k^{2}}{\cosh ^{2} k t}\right)
\end{aligned}
$$


where $a_{0}$ and $a_{3}$ are constants of integration. For a massive particle $(m=1), 3$-velocity with respect to an observer $Z=(1 / f) \partial_{t}$ is given by

$$
v^{2}=1-\left\{a_{0} / f^{2}+\left(a_{3} k / f \cosh k t\right)^{2}\right\}^{-1}
$$

For any given domain wall solution these equations can be solved numerically. We will work with $f(x)=\cosh ^{-q}(k x / q)[(3.22)]$ solution but the essential features of particle motion remain unchanged in other spacetimes obtained from (3.19)-(3.21). Since $f(x)$ decreases monotonically away from $x=0$ (where it attains its unique maximum), the 3 -velocity of the partcle with respect to the observer $Z$, will increase away from $x=0$. This shows the repulsive nature of the domain wall gravitational field

Since domain wall spacetimes allow repulsive gravity it is clear that "turning point" solutions in which a incoming particle is repelled by the domain wall are allowed. The phase space plot for one such geodesic is shown in figure 10. Since the spacetime is expanding and particles are accelerated away from the wall the coordinate time $t$ along a geodesic is red shifted. A plot of $t(\tau)$ is shown in figure 11.

\section{B. Static Spacetimes}

To analyse particle motion in a static domain wall spacetime we now consider the geodesic equations for a test particle (of mass $m=0,1$ ). Such a particle may be represented by a curve on $\left(M^{4}, g\right)$ [with $g$ given by (4.15) and (4.25)-(4.27)] :

$$
\gamma: \tau \mapsto(t=t(\tau), \xi=\xi(\tau), y=y(\tau), z=z(\tau))
$$


If $\gamma$ is a geodesic (and $\tau$ is an affine parameter along $\gamma$ ), then the 4 -velocity of the particle is

$$
\gamma_{*}=\left(\frac{d t}{d \tau}\right) \partial_{t}+\left(\frac{d \xi}{d \tau}\right) \partial_{\xi}+\left(\frac{d y}{d \tau}\right) \partial_{y}+\left(\frac{d z}{d \tau}\right) \partial_{z}
$$

with the normalisation $g\left(\gamma_{*}, \gamma_{*}\right)=-m^{2}$ :

$$
-F^{2}\left(\frac{d t}{d \tau}\right)^{2}+N^{2}\left(\frac{d \xi}{d \tau}\right)^{2}+(N / F)\left\{\left(\frac{d y}{d \tau}\right)^{2}+\left(\frac{d z}{d \tau}\right)^{2}\right\}=-m^{2}
$$

The geodesic equations are given by

$$
\begin{aligned}
\frac{d t}{d \tau} & =C_{0} / F^{2} \\
\frac{d}{d \tau}\left(N^{2} \frac{d \xi}{d \tau}\right) & =\frac{1}{2}\left\{-\left(F^{2}\right)^{\prime}\left(\frac{d t}{d \tau}\right)^{2}+\left(N^{2}\right)^{\prime}\left(\frac{d \xi}{d \tau}\right)^{2}+(N / F)^{\prime}\left(\frac{d y}{d \tau}\right)^{2}+(N / F)^{\prime}\left(\frac{d y}{d \tau}\right)^{2}\right\} \\
\frac{d y}{d \tau} & =C_{2} /(N / F) \\
\frac{d z}{d \tau} & =C_{3} /(N / F)
\end{aligned}
$$

where $C_{0}, C_{2}$, and $C_{3}$ are constant and 'prime' represents differentiation with respect to $\xi$.

For a massive particle $(m=1)$, the 3 -velocity with respect to an observer $Z=(1 / F) \partial_{t}$ is given by

$$
v^{2}=1-\left(F / C_{0}\right)^{2}
$$

Since $F$ is a monotonically decreasing positive function, a massive particle that starts on the left side with a negative velocity will move away from the wall, reach a turning point and be accelerated back towards the wall. After the particle has passed through the wall it will be repelled from the wall. Thus, on the left side of the domain wall (Taub-vacuum), gravity is attractive while, on the right side (Minkowsky-vacuum), gravity is repulsive. A phase space plot for such a particle is shown in figure 12 . 
For a photon traveling along $\xi$-axis, the corresponding null geodesic is represented by

$$
\gamma: \lambda \mapsto(t=t(\lambda), \xi=\xi(\lambda), y=a, z=b)
$$

where, $\lambda$ ia an affine parameter, and $a, b$ are constants. This null geodesic is characterised by

$$
\begin{aligned}
\gamma_{*} & =\left(\frac{d t}{d \lambda}\right) \partial_{t}+\left(\frac{d \xi}{d \lambda}\right) \partial_{\xi} \\
\frac{d t}{d \lambda} & =C_{0} / F^{2} \\
g\left(\gamma_{*}, \gamma_{*}\right) & =0=-F^{2}\left(\frac{d t}{d \lambda}\right)^{2}+N^{2}\left(\frac{d \xi}{d \lambda}\right)^{2}
\end{aligned}
$$

Now, with respect to the observer field $Z=(1 / F) \partial_{t}$, we compute the frequency ratio for the photons traveling along $\xi$-axis:

$$
\frac{f_{e}}{f_{o}}=\left\{-g\left(\gamma_{*}, Z_{e}\right)\right\} /\left\{-g\left(\gamma_{*}, Z_{o}\right)\right\}=F\left(\xi_{o}\right) / F\left(\xi_{e}\right)
$$

where $e$ and $o$ are emission-point and observation-point respectively. From the above equation, we note that photons approaching the wall from the left-hand (Taub) side will be continuously blue-shifted as they pass through the wall toward the Minkowski vacuum. Photons that approach the wall from the right-hand (Minkowski) side of the wall will first be red-shifted and then blue-shifted when they pass through the wall into the Taub side.

The geodesic equations with respect to the generalised static wall metric discussed in section 4.5, can be obtained by replacing $F$ in the above equations. The essential characteristics of particle motion are the same as discussed above. 


\section{CONCLUSIONS}

We have found new classes of static and non-static domain wall solutions to the coupled Einstein-scalar field equations which are (locally) plane symmetric. The static walls are particularly interesting since static domains walls cannot be reflection symmetric. Consequently, static domain walls must possess different asymptotic vacua. In principle, it should be possible to detect static domain walls by studing the red-shift of photons. The fact that photons approaching the wall from the Taub side are continuously blue-shifted as they pass through the wall toward the Minkowski vacuum, while photons that approach the wall from the Minkowski side of the wall are first red-shifted and then blue-shifted when they pass through the wall into the Taub side provides a unique experimental signature.

It is generally believed that small perturbations in the mass distribution in the early Universe could subsequently grow [20,21] to form galaxies and the structures we currently see around us. However, one of the major problems [22] in modern cosmology is to identify the origin of these perturbations. The domain walls discussed in this paper could be possible sources of the density fluctuations through their specific gravitational interactions with the ambient matter.

\section{Acknowledgement}

MM would like to thank Mark Jarrell and Frank Pinski for their help where it counts, Louis

Witten, Paul Esposito and Rohana Wijewardhana for their interest in this work, and Robin Endelman for many helpful discussions. RG would like to thank Claudia Taylor for helpful discussions. 


\section{APPENDIX}

Lemma 1: $\left(u^{\prime}+2 v^{\prime}\right)<0$ [Equation (4.5)].

Proof: Equating (4.2) and (4.4) leads to

$$
2\left(v^{2}-u v\right)=u^{\prime}-v^{\prime}
$$

Inserting the above relation in (4.3) gives

$$
u^{\prime}+v^{\prime} / 2=-(2 \pi G) f^{2}(3 \rho+\nu)<0
$$

This equation, together with $v^{\prime}<0$ [which follows from (4.4)], proves the lemma.

Lemma 2: Let $\nu(x)$ be a smooth nowhere-zero function on $\mathbb{R}$. If $\lim _{|x| \rightarrow \infty} \nu=0$, then there is an $\bar{x}$ such that $\nu^{\prime}(\bar{x})=0$.

Proof: Suppose that $\nu^{\prime} \neq 0$ for all $x \in \mathbb{R}$. Since $\nu(x)$ is smooth, $\nu^{\prime}(x)$ is continuous and hence, either $\nu^{\prime}(x)>0$ or $\nu^{\prime}(x)<0$. If $\nu^{\prime}(x)>0$, then $\nu$ is a strictly increasing function on $\mathbb{R}$, and hence it can not satisfy the boundary condition $\lim _{|x| \rightarrow \infty} \nu=0$. For $\nu^{\prime}(x)<0$, a similar argument leads to the same contradiction. Thus, there exists an $\bar{x} \in \mathbb{R}$ such that $\nu^{\prime}(\bar{x})=0$. 


\section{REFERENCES}

[1] T. W. B. Kibble, J. Phys. A: Math. Gen. 91387 (1976).

[2] Y. B. Zel'dovich, I. Y. Kobzarev and L. B. Okun, Zh. Eksp. Teor. Fiz. 673 (1974). (Sov. Phys.- JETP 401 (1975))

[3] M. Cvetič and H. Soleng,Phys. Rep. 282159 (1997).

[4] I.A. Vilenkin, Phys. Rep. 121263 (1985).

[5] M. F. Parry and A. T. Sornborger, Domain Wall Production During Inflationary Reheating, hep-ph/9805211 Preprint (1998)

[6] A. Albrecht, D. Coulson, P. Ferreira and J. Magueijo, PRL. 761413 (1996).

[7] C. T. Hill, D. N. Schramm and J. N. Fry, Commun Nucl. Part. Phys. 1925 (1989).

[8] M. Mukherjee, Class. Quatum Grav., 10131 (1993).

[9] A. Vilenkin, Phys. Lett. 133B 177 (1983).

[10] G. Goetz, J. Math. Phys. 312683 (1990), L. M. Widrow, Phys. Rev. D39 3571 (1989), J. Isper and P. Sikivie, Phys. Rev. D30 712 (1984).

[11] Mirjam Cvetič, Richard L. Davis, Stephen Griffies, and Harald H. Soleng. Phys. Rev. Lett., 70(9):1191-1194, March 1993.

[12] Mirjam Cvetič, Stephen Griffies, and Harald H. Soleng. Phys. Rev. Lett., 71(5):670-673, August 1993.

[13] Mirjam Cvetič, Stephen Griffies, and Harald H. Soleng. Phys. Rev. D, 48(6):2613-2634, September 1993. 
[14] Mirjam Cvetič and Harald H. Soleng. Phys. Rev. D, 51(10):5768-5784, May 1995.

[15] Bjørn Jensen and Harald H. Soleng. Class. Quant. Grav., 14:1821-1829, 1997.

[16] A. D. Dolgov and I. B. Khriplovich. Gen. Relativ. Gravit., 21(1):13-16, 1989.

[17] B. Linet. Int. J. Theor. Phys., 24(12):1159-1164, 1985.

[18] A. H. Taub, Ann. Math. 53472 (1951).

[19] R. K. Sachs and H.Wu, General Relativity for Mathematicians, Springer-Verlag (1977).

[20] D. H. Lyth and M. Mukherjee, Phys. Rev. D38 485 (1988).

[21] R. H. Brandenberger, J. Phys. G15 1 (1989).

[22] P.J.E. Peebles, Principles of Physical Cosmology, Princeton University Press (1993). 


\section{FIGURES}

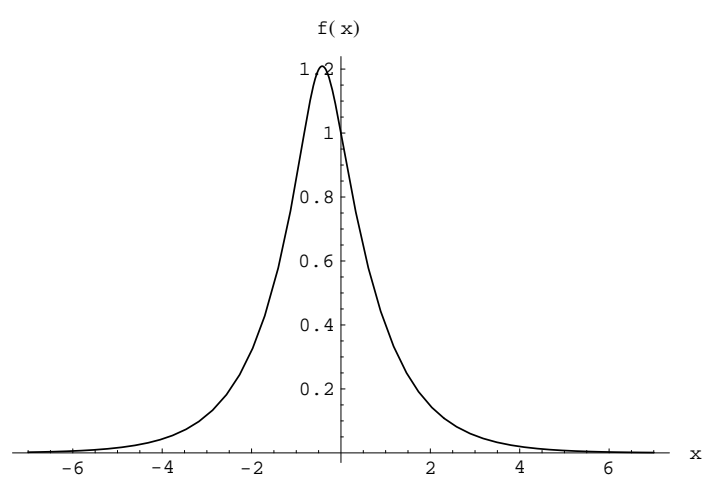

FIG. 1. Plot of $f(x)$ as defined by Eq. 3.29 versus $x$. Note that the domain wall is no longer centered at $x=0$.

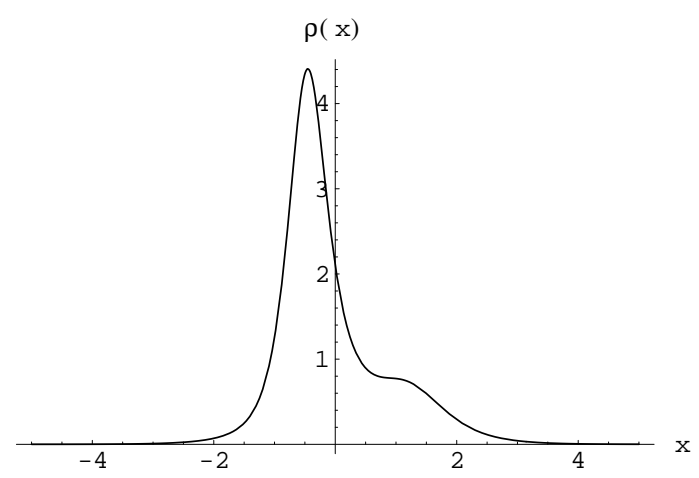

FIG. 2. Plot of the energy density $\rho(x)$ versus $x$ for $k=1$.

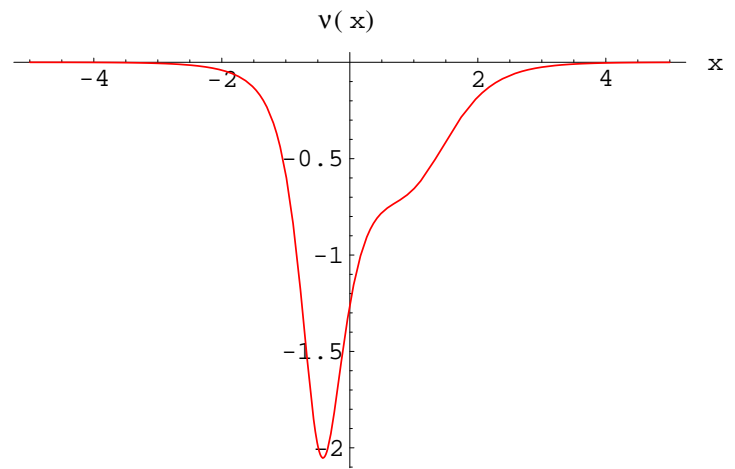

FIG. 3. Plot of the pressure $\nu(x)$ versus $x$ for $k=1$. Note that the pressure is negative, as it must be for a domain wall solution. 


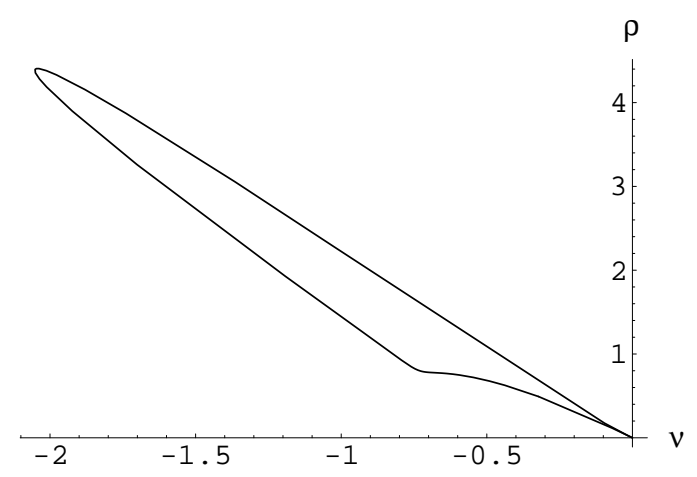

FIG. 4. Plot of the energy density $\rho(x)$ versus the pressure $\nu(x)$ for $k=1$.

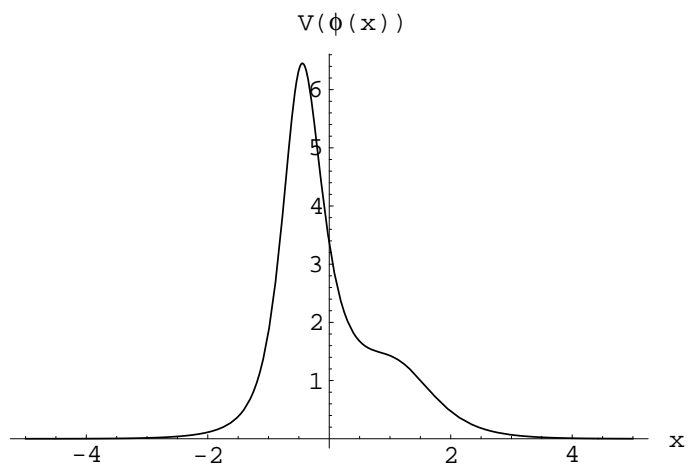

FIG. 5. Plot of the potential $V(\phi)$ versus $x$ for $k=1$.

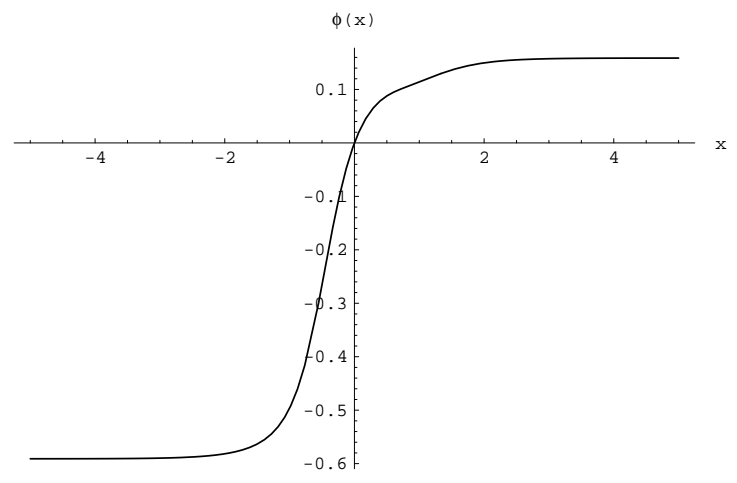

FIG. 6. Plot of the field $\phi(x)$ versus $x$ for $k=1$. 


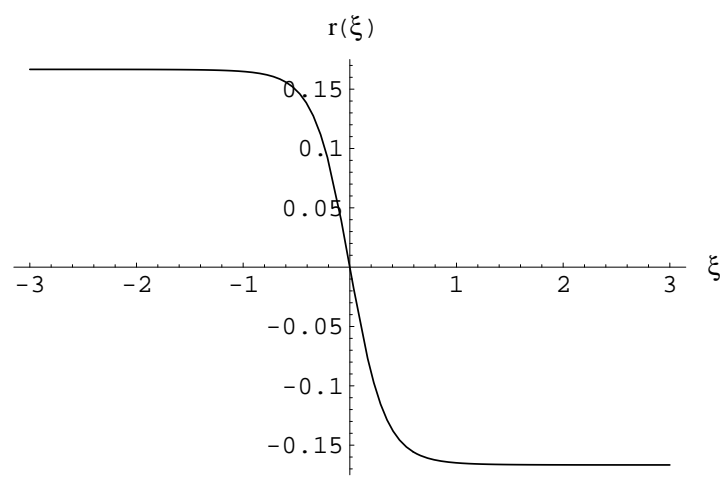

FIG. 7. Plot of $r(\xi)$ as defined by Eq. 4.46 versus $x$ for $K=1$.

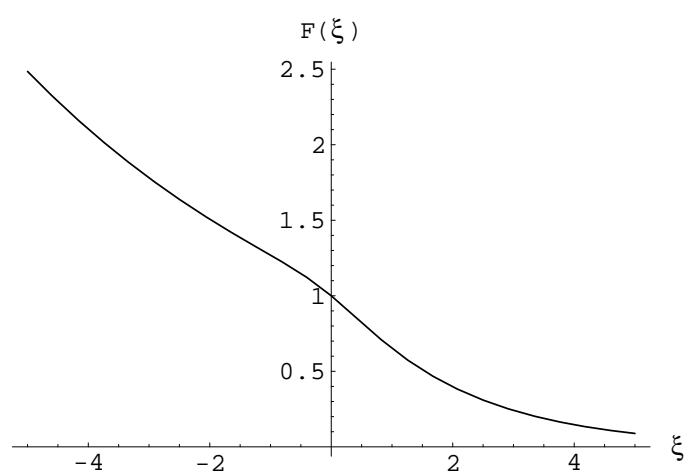

FIG. 8. Plot of $F(\xi)$ versus $\xi$ for $K=1$.

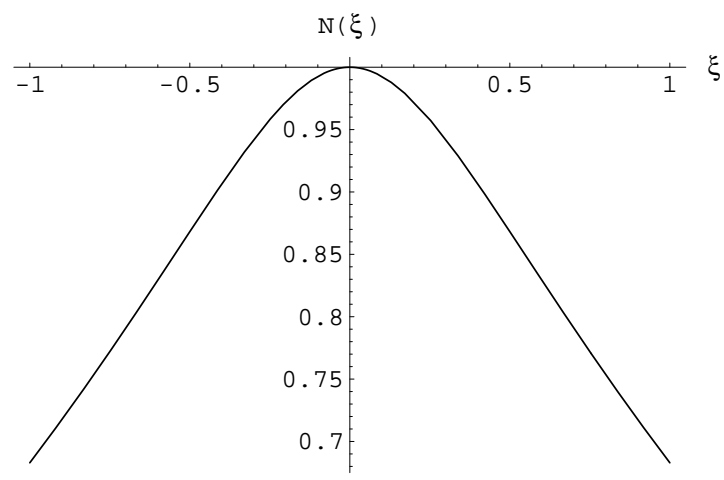

FIG. 9. Plot of $N(\xi)$ versus $\xi$ for $K=1$. 


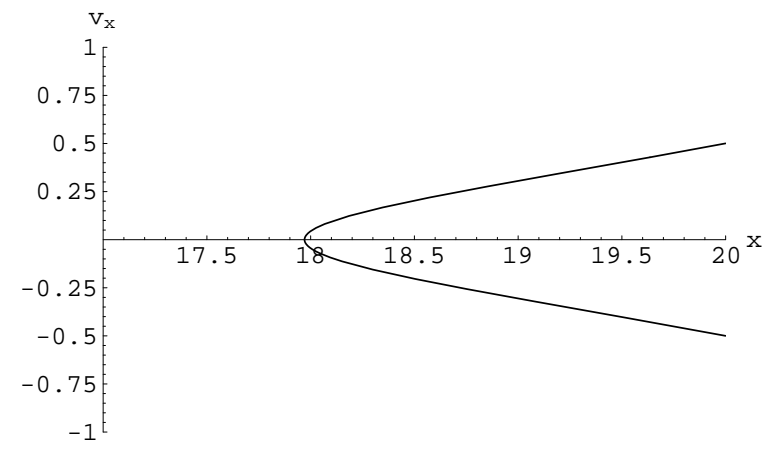

FIG. 10. Phase space plot of a turning point solution for a particle with initial conditions $x(0)=20, x^{\prime}(0)=-0.5, y^{\prime}(0)=0.5, y(0)=0, z^{\prime}(0)=0, z(0)=0, t^{\prime}(0)=1, t(0)=0$ and $\mathrm{f}$ given by 3.22 .

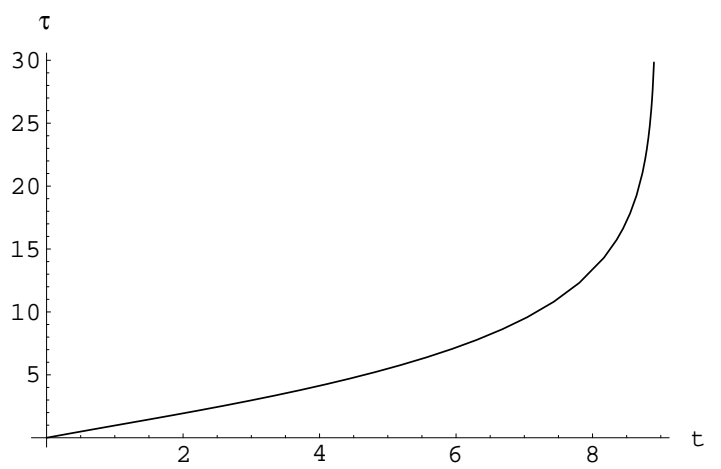

FIG. 11. A plot of $\tau(t)$ showing the redshift of the $t$ for a particle with initial conditions $x(0)=1 / 2, x^{\prime}(0)=-0.5, y^{\prime}(0)=0, y(0)=0, z^{\prime}(0)=0, z(0)=0, t^{\prime}(0)=1, t(0)=0$ and $\mathrm{f}$ given by 3.22 .

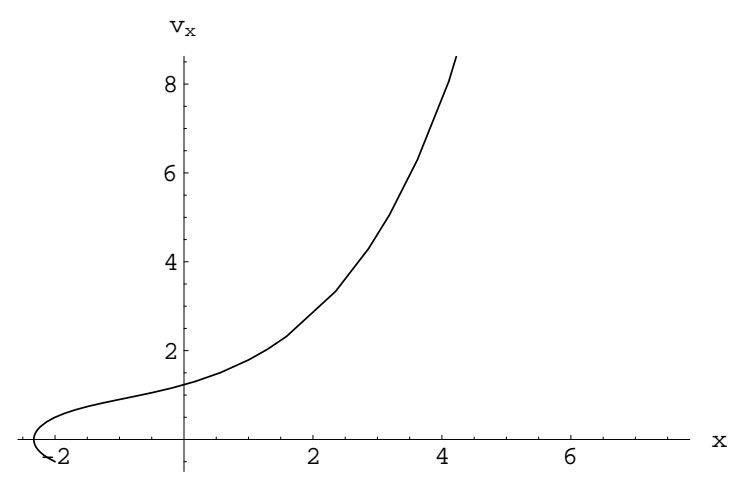


FIG. 12. Phase space plot of a turning point solution for a unit mass particle with initial conditions $x(0)=2, x^{\prime}(0)=-0.5, y^{\prime}(0)=0.9188, y(0)=0, z^{\prime}(0)=0, z(0)=0, t^{\prime}(0)=1, t(0)=0$. On the left hand side of the domain wall gravity is attractive, while on the right hand side of the domain wall gravity is repulsive. The space-time has parameters $m=1, L=1 / 2$, and $k=1 / 2$ and metric given by $g$ in $(4.15$ and $4.25-4.27)$

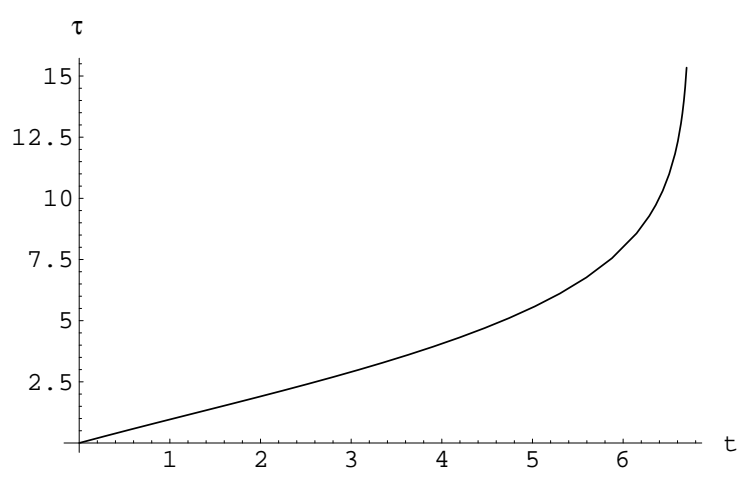

FIG. 13. The red shift of $\tau(t)$ along a gedodesic for a unit mass particle with initial conditions $x(0)=2, x^{\prime}(0)=-0.5, y^{\prime}(0)=0.9188, y(0)=0, z^{\prime}(0)=0, z(0)=0, t^{\prime}(0)=1, t(0)=0$. The space-time has parameters $m=1, L=1 / 2$, and $k=1 / 2$ and metric given by $g$ in $(4.15$ and $4.25-4.27)$ 